\title{
Correction to: A missense mutation in IFT74, encoding for an essential component for intraflagellar transport of Tubulin, causes asthenozoospermia and male infertility without clinical signs of Bardet-Biedl syndrome
}

Patrick Lorès ${ }^{1} \cdot$ Zine-Eddine Kherraf $^{2,3} \cdot$ Amir Amiri-Yekta $^{4} \cdot$ Marjorie Whitfield $^{2} \cdot$ Abbas Daneshipour $^{4}$. Laurence Stouvenel $^{1}$. Caroline Cazin ${ }^{2,3} \cdot$ Emma Cavarocchi $^{2} \cdot$ Charles Coutton $^{2,5} \cdot$ Marie-Astrid Llabador $^{6}$. Christophe Arnoult ${ }^{2}$. Nicolas Thierry-Mieg ${ }^{7}$. Lucile Ferreux ${ }^{1,8}$. Catherine Patrat ${ }^{1,8}$. Seyedeh-Hanieh Hosseini ${ }^{9}$.

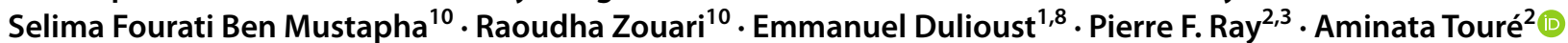

Published online: 26 March 2021

(c) Springer-Verlag GmbH Germany, part of Springer Nature 2021

\section{Correction to: Human Genetics} https://doi.org/10.1007/s00439-021-02270-7

In the original article published, the family name of the second author is incorrect. The correct name is Zine-Eddine Kherraf.

The original article has been corrected.

The original article can be found online at https://doi.org/10.1007/ s00439-021-02270-7.

Aminata Touré

aminata.toure@inserm.fr

1 Université de Paris, Institut Cochin, INSERM, CNRS, 75014 Paris, France

2 Université Grenoble Alpes, Institut pour l'avancée des Biosciences, INSERM, CNRS, 38000 Grenoble, France

3 CHU de Grenoble, UM GI-DPI, 38000 Grenoble, France

4 Department of Genetics, Reproductive Biomedicine Research Center, Royan Institute for Reproductive Biomedicine, ACECR, Tehran, Iran

5 CHU Grenoble Alpes, UM de Génétique Chromosomique, Grenoble, France

6 Laboratoire de Biologie de la Reproduction, Groupe Hospitalier Universitaire Paris Nord Val de Seine, Assistante Publique-Hôpitaux de Paris, 75018 Paris, France
Publisher's Note Springer Nature remains neutral with regard to jurisdictional claims in published maps and institutional affiliations.
7 Univ. Grenoble Alpes, CNRS, TIMC-IMAG/BCM, 38000 Grenoble, France

8 Laboratoire d'Histologie Embryologie, Biologie de la Reproduction, CECOS Groupe Hospitalier Universitaire Paris Centre, Assistance Publique-Hôpitaux de Paris, 75014 Paris, France

9 Department of Andrology, Reproductive Biomedicine Research Center, Royan Institute for Reproductive Biomedicine, ACECR, Tehran, Iran

10 Polyclinique les Jasmins, Centre d'Aide Médicale à la Procréation, Centre Urbain Nord, 1003 Tunis, Tunisia 